Musées, Patrimoine et Culture scientifiques et techniques

$168 \mid 2016$

novembre-décembre 2016

\title{
L'impact des collections patrimoniales sur la santé
}

\section{Laurent Defendini}

URL : http://journals.openedition.org/ocim/1708

DOI : $10.4000 /$ ocim. 1708

ISSN : 2108-646X

\section{Éditeur}

OCIM

Édition imprimée

Date de publication : 1 novembre 2016

Pagination : 12-15

ISSN : 0994-1908

Référence électronique

Laurent Defendini, «L'impact des collections patrimoniales sur la santé », La Lettre de I'OCIM [En ligne], 168 | 2016, mis en ligne le 01 novembre 2017, consulté le 20 avril 2019. URL : http://

journals.openedition.org/ocim/1708; DOI : 10.4000/ocim.1708

Ce document a été généré automatiquement le 20 avril 2019

Tous droits réservés 


\title{
L'impact des collections patrimoniales sur la santé
}

\author{
Laurent Defendini
}

Capteur de radon posé sur une ammonite dans les réserves de paléontologie de la Galerie de Paléontologie et d'Anatomie comparée.

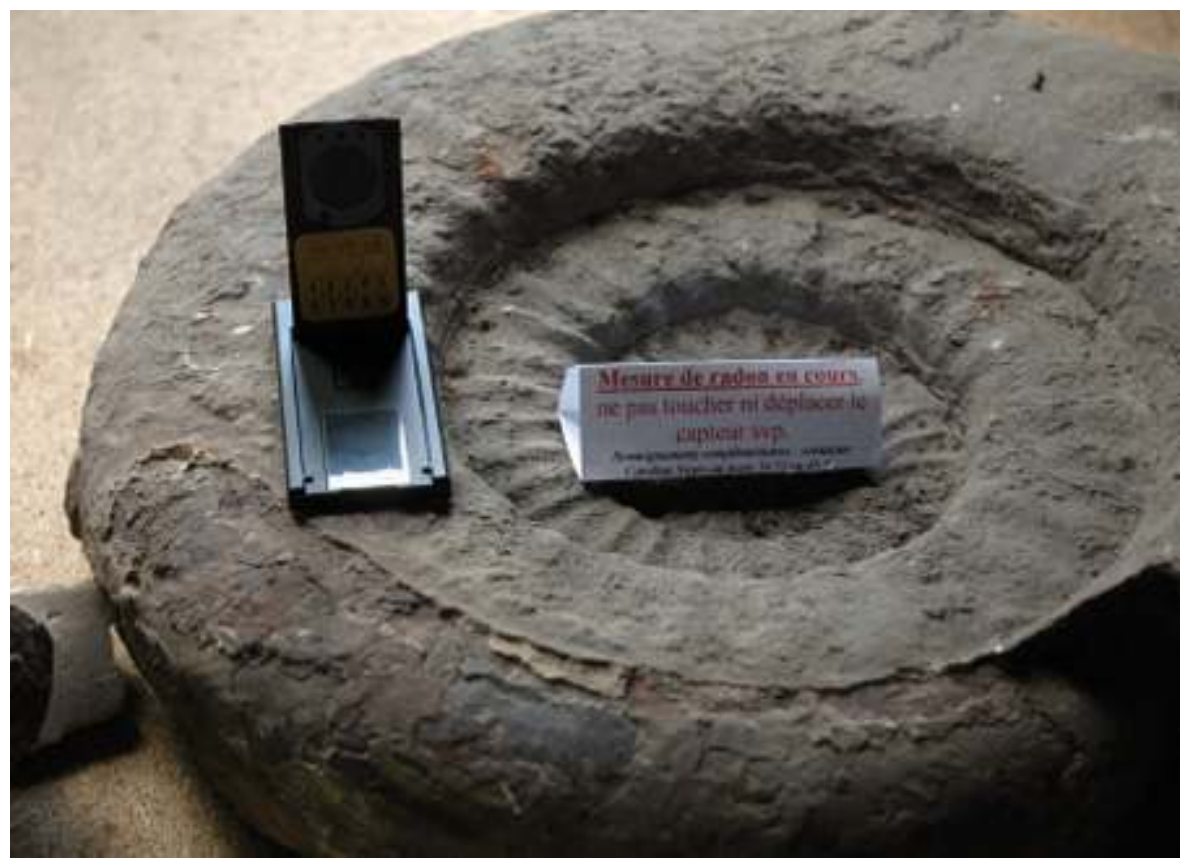

(c) MNHN

\section{Le MNHN et la direction des collections}

Le Muséum national d'Histoire naturelle (MNHN) est un établissement public d'État sous une double tutelle : le ministère de l'Éducation nationale, de l'Enseignement supérieur et 
de la Recherche et le ministère de l'Environnement, de l'Énergie et de la Mer. Environ 170 agents travaillent pour la direction des collections sur 1700 que compte le MNHN. 26 bâtiments au total sont concernés par les collections, situés principalement sur le site du Jardin des Plantes, incluant aussi le musée de l'Homme, ainsi qu'un entrepôt situé en banlieue parisienne. Ces locaux comprennent bureaux, ateliers, laboratoires et réserves de collections. Les 68 millions d'objets ou de spécimens (estimation) du MNHN occupent environ $30000 \mathrm{~m}^{2}$.

2 Pour mener sa politique de prévention, la direction des collections privilégie l'aspect préventif à l'aspect curatif. Une ligne budgétaire spécifique "hygiène et sécurité" (H \& S) a été créée. Les agents font l'objet d'une sensibilisation et d'une pédagogie à travers des formations $\mathrm{H} \& \mathrm{~S}$ spécifiques et des analyses des accidents et presqu'accidents au quotidien. Les presqu'accidents, notion nouvelle, sont principalement des incidents matériels n'ayant fait aucune victime mais auraient pu être dramatiques, si cela avait touché directement des agents.

3 La difficulté rencontrée sur la prévention dans le domaine des collections patrimoniales est de réussir à trouver le juste milieu entre la contrainte due aux expositions aux risques professionnels des agents et la déontologie propre aux collections, notamment relative à la conservation et à la conservation préventive.

4 Pour illustrer ces propos, prenons l'exemple des collections naturalistes en fluide conservateur tel que le formaldéhyde (plus communément appelé formol). Il est classé substance Cancérogène, Mutagène et toxique pour la Reproduction (CMR), produit chimique considéré comme dangereux. La règlementation nous impose de ne plus utiliser de CMR mais des produits de substitution, recommandation portée par les conseillers de prévention dans les établissements de l'enseignement supérieur et de la recherche. Or, encore aujourd'hui, il n'existe aucun produit de substitution au formol.

5 Certaines collections peuvent se retrouver déformées accidentellement par leur passage du formol à l'éthanol, c'est le cas des collections de certains invertébrés marins tels que les ascidies et certains cnidaires, ou des foetus de vertébrés.

6 La seule solution aujourd'hui, est donc de doter les lieux où il peut y avoir manipulation $\mathrm{du}$ formol d'équipements adéquats d'aspiration (hottes et bras à extraction) permettant de limiter l'exposition des agents aux vapeurs de formol. Le tout complété par la mise à disposition d'équipements de protection individuels adaptés (masques de protection respiratoire avec cartouches filtres).

7 Rappelons qu'en matière de prévention des risques professionnels, il faut adapter l'environnement du travail à l'homme et non l'inverse.

\section{Quelques exemples de collections patrimoniales à risques spécifiques}

Les collections entomologiques

9 Les risques professionnels de cette collection sont identifiés et bien connus. Il y a la rémanence des insecticides toxiques utilisés par nos prédécesseurs (exemple du lindane). On utilise également un colorant toxique ; le noir de chlorazol pour la coloration des genitalia (produit chimique classé CMR). 
10 La politique de prévention préconise la séparation des bureaux (postes de travail) des espaces de réserves de collections, et le remplacement du noir de chlorazol par un colorant non toxique.

\section{Les collections paléontologiques et minéralogiques radioactives}

La radioactivité est un risque professionnel non palpable, inodore et incolore. Il nécessite une identification des spécimens géologiques ou paléontologiques contaminés avec des appareils de mesure adaptés à la mesure de la radioactivité comme les compteurs Geiger.

Certains minéraux présentent une radioactivité naturelle parfois très importante, et dans une mesure nettement moindre, certains fossiles également. Cette radioactivité génère un risque d'effets stochastiques comme les effets de faibles doses de toxiques ou les effets des rayonnements ionisants néfastes à la santé, difficilement évaluable, en cas d'exposition fréquente et prolongée.

Les risques professionnels identifiés concernent l'émission naturelle de rayonnements ionisants et de radon, un gaz radioactif toxique.

14 La politique de prévention suppose aujourd'hui un audit des collections de paléontologie et de minéralogie par une entreprise extérieure spécialisée pour réaliser un inventaire précis des spécimens radioactifs.

Signalement de la présence de spécimens radioactifs à risques dans les réserves de la Grande Galerie de Minéralogie

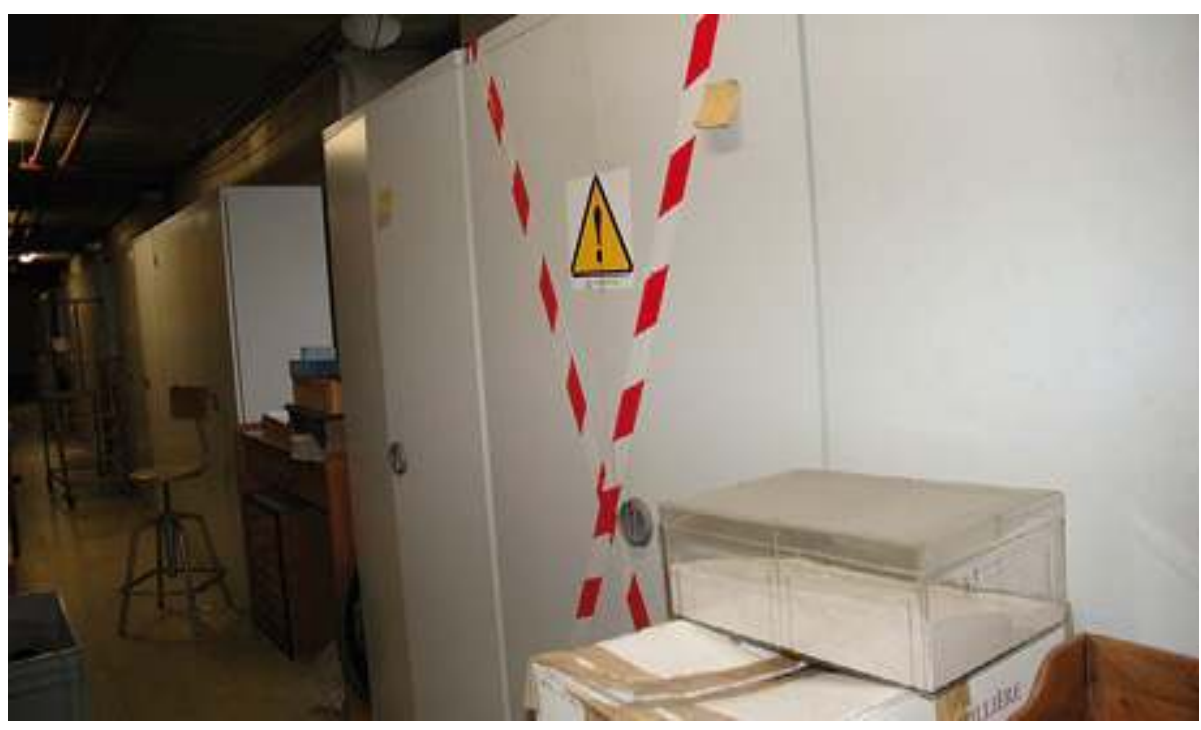

(C) MNHN

15 Il faut séparer les spécimens radioactifs de la collection générale et les entreposer dans un lieu protégé (armoires plombées). Il faut également un affichage des pictogrammes "risques radioactifs" dans les réserves, pour signaler la présence de spécimens radioactifs à risques et mettre en place des dosimètres individuels pour les agents principalement exposés. Enfin, il faut une vérification trimestrielle des spécimens et de l'environnement par des appareils de mesures spécialisés (radiamètre, contaminomètre, capteur de radon). 


\section{Les collections en fluide (alcool / formol)}

Collections d'ichtyologie à la zoothèque (conditionnement en tonneaux)

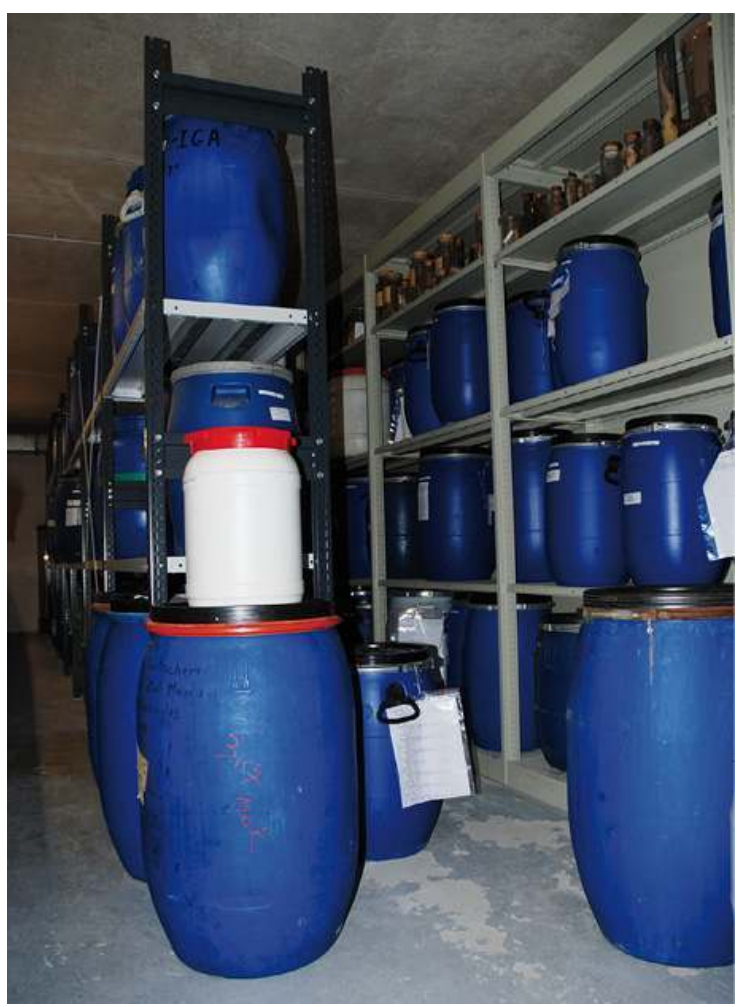

(c) $\mathrm{MNHN}$ 


\section{Les collections de spécimens de vertébrés naturalisés}

\section{aujourd'hui, en dehors de l'utilisation des équipements de protection individuels} classiques tels que des gants, masques anti-poussière, blouses fermées.

\section{Santé, sécurité et prévention des risques professionnels dans les collections patrimoniales}

L'atteinte à la santé d'un salarié dans un cadre professionnel est due aux expositions aux risques professionnels. L'hygiène et la sécurité dans le domaine des collections patrimoniales peuvent se décliner en quatre volets : 
32 - la prévention

33 - l'identification des risques professionnels

34 - les analyses des accidents et presqu'accidents

\section{Les obligations réglementaires en quelques exemples}

Il est nécessaire qu'il y ait un suivi de l'évacuation des déchets (chimiques, biologiques, radioactifs et autres), ainsi que le Document Unique d'Évaluation des Risques (DUER) accompagné de son programme annuel de prévention du service.

Concernant la ville de Paris, un Plan de Protection contre les Inondations (PPcI) doit être élaboré, en prévision d'une potentielle crue centennale de la Seine.

Des fiches simplifiées d'exposition aux risques professionnels, avec mise à jour annuelle permettant de faire le lien entre l\&apos;activité professionnelle et l'apparition d'une nouvelle pathologie, doivent être établies.

Les vérifications annuelles sont par ailleurs obligatoires : extincteurs et détecteurs d'incendie, alarmes sonores et visuelles, sorbonnes, installations électriques des locaux et des équipements, des appareils ou des objets émettant des rayonnements ionisants, des appareils de levage et des paletiers...

\section{La prévention}

39 Les agents doivent porter des équipements de protection individuels (EPI), tels les gants, lunettes de protections, blouses, masques, et les lieux dédiés doivent comporter des équipements de protection collectifs (EPC), comme les sorbonnes, les hottes à filtration.

40 Il faut des formations H \& S (manipulation d'extincteur, risques chimiques...) et faire respecter les règles de sécurité en fonction des risques inhérents à l'activité, par exemple lors des missions de terrain : rappel des procédures de sécurité dans le cadre de collectes de spécimens sur le terrain...

\section{L'identification des risques professionnels}

41 Les principaux risques professionnels dans les collections patrimoniales peuvent être classés selon sept rubriques : électrique, chimique, biologique, radioactif, incendie, manutention (port de charges lourdes entrainnant des troubles musculo-squelettiques), risques psychosociaux.

\section{Les accidents et presqu'accidents}

42 L'organisation des premiers secours (secouristes salariés formés, procédure en lien avec les services de sécurité...) est essentielle.

43 La tenue des registres H \& S (deux types de registres : Santé et sécurité au travail et Dangers graves et imminents) doit être continue et sérieuse. Il doit exister un lien automatique avec la médecine de prévention, infirmerie, pompiers. L'expérience tirée des analyses des accidents et presqu'accidents doit servir. 
Quartz fumé de $830 \mathrm{~kg}$ de l'exposition permanente de minéralogie à la Grande Galerie de Minéralogie

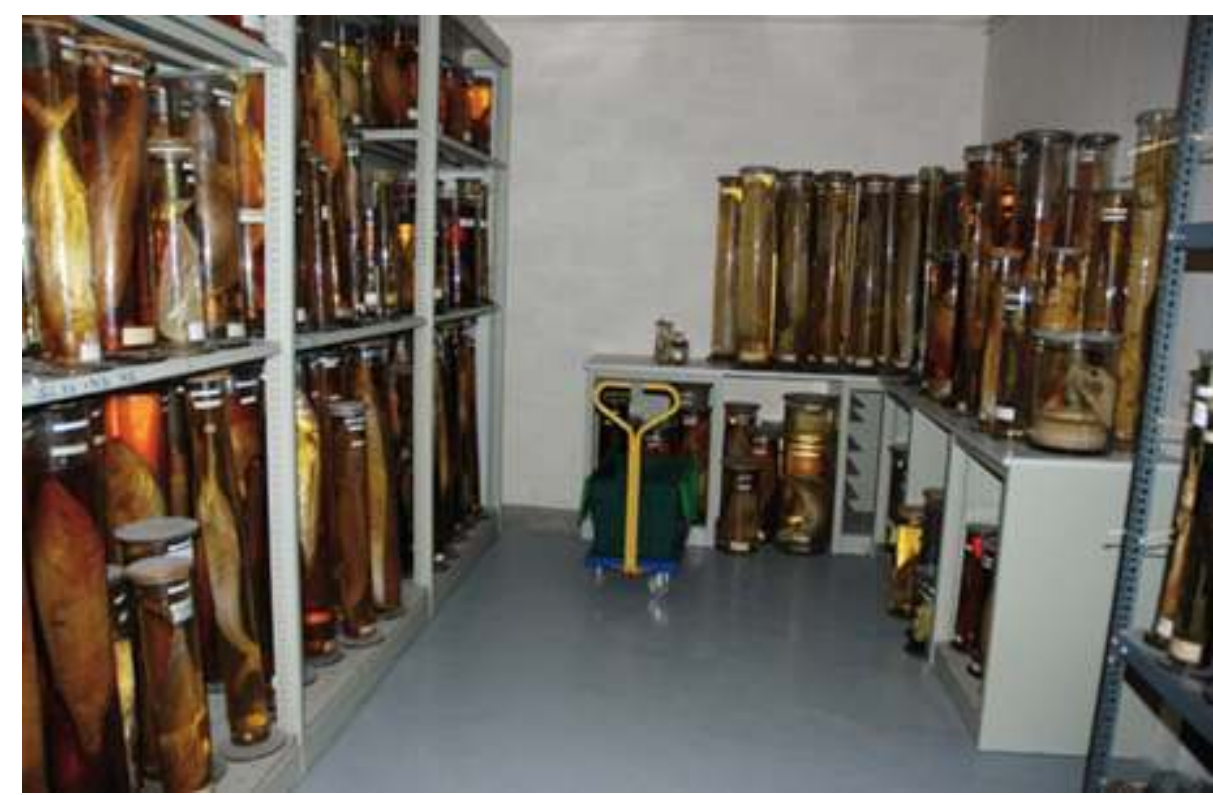

(c) MNHN

\section{Les solutions...} à jour), dont voici quelques exemples : travail avec blouse fermée obligatoire, lunettes de protection obligatoires si travail sur fluide, mise en place d'équipements de protection collectifs (EPC), à défaut stockage et mise à disposition d'équipements de travail individuels adaptés ;

47 pedagogie et une sensibilisation permanentes des agents aux risques professionnels de leur poste de travail à travers des formations individuelles adaptées à leurs activités, avec sensibilisation aux risques professionnels propres au poste de travail et son environnement ; 


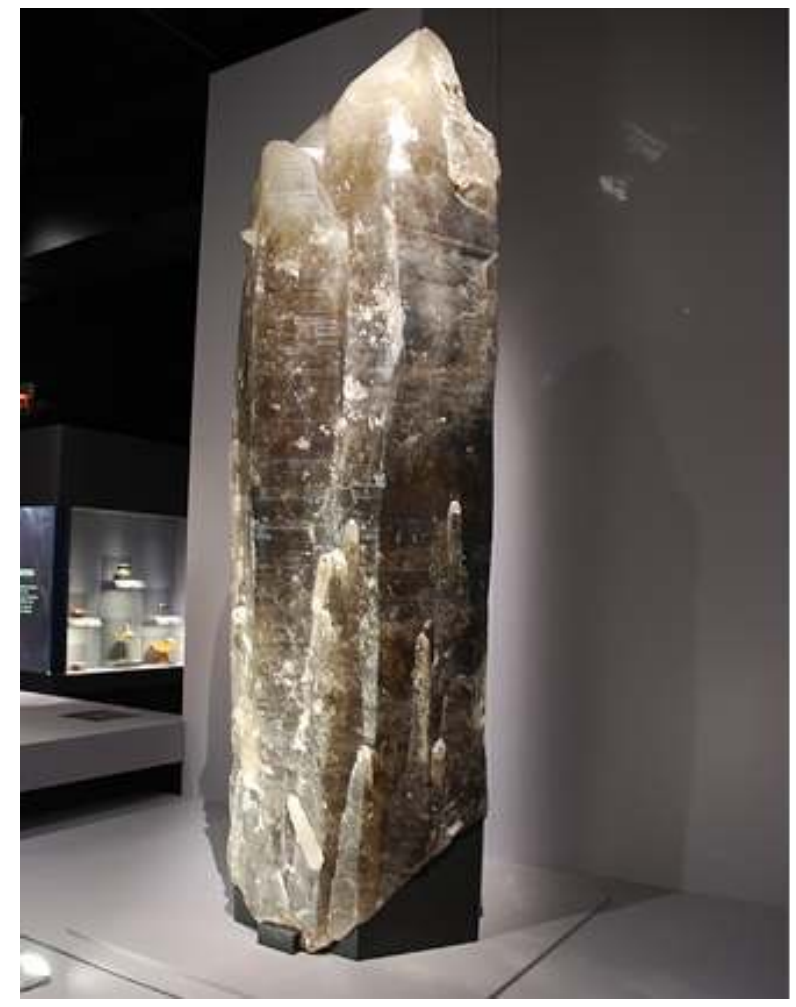

(c) MNHN

Dans le cas de la fonction publique territoriale, il ne faudra pas hésiter à s'appuyer sur les acteurs de la prévention tel que le médecin du travail, dont le rôle consiste à prévenir toute altération de la santé du fait du travail, contribuer à l'évaluation des risques, contribuer aux actions de prévention, de correction ou d'amélioration des conditions de travail et effectuer des études de poste et des visites de locaux de travail.

\section{Conclusion}

La prévention à la direction des collections du MNHN n'est pas destinée à protéger ou limiter les expositions aux risques professionnels uniquement pour les agents de la direction des collections, mais aussi l'ensemble des personnes susceptibles d'être exposées lors de la consultation de collections dans nos réserves.

51 Ces personnes consultant les collections au MNHN sont principalement des enseignantschercheurs, des étudiants, des doctorants et des professeurs d'universités appartenant à l'établissement, mais également les collègues scientifiques étrangers et autres divers visiteurs venant également aussi consulter les collections.

52 Attention, il ne faut pas aussi oublier dans les personnels potentiellement exposés, les autres catégories de personnel, tels que les agents des services généraux comme ceux de la sécurité, du nettoyage, de la rénovation ainsi que les entreprises extérieures intervenant ponctuellement dans les locaux de collections (ateliers, réserves...). 


\section{RÉSUMÉS}

L'exemple des collections du Muséum national d'Histoire naturelle et l'identification des risques pour la santé encourus par les personnels lors des opérations de manipulation et de conservation préventive des spécimens, permettent à l'auteur de préciser les règles d'hygiène et de sécurité indispensables pour mener à bien une bonne politique de prévention.

INDEX

Mots-clés : hygiène, sécurité, collection

\section{AUTEUR}

\section{LAURENT DEFENDINI}

référent hygiène et sécurité à la Direction des collections au Muséum national d'Histoire naturelle et responsable adjoint de la zoothèque

laurent.defendini@mnhn.fr 